\title{
Evaluación de metales pesados en los sedimentos superficiales del río Pirro
}

\author{
Evaluation of heavy metals in superficial \\ sediments of Pirro river
}

Jacqueline Herrera Núñez'

José Rodríguez Corrales ${ }^{2}$ Juana María Coto Campos ${ }^{3}$

Viviana Salgado Silva ${ }^{4}$

Henry Borbón Alpizar ${ }^{5}$

Fecha de recepción: 14 de diciembre del 2011 Fecha de aprobación: I de octubre del 2012

Herrera, j; Rodríguez, J; Coto, J; Salgado, V; Borbón, H. Evaluación de metales pesados en los sedimentos superficiales del río Pirro Tecnología en Marcha. Vol. 26, № I. Pág 27-36 


\section{Palabras clave}

Río Pirro, metales pesados, sedimentos.

\section{Resumen}

Los metales pesados tienen una importante fuente en las actividades de origen antropogénico y constituyen un peligro para la biota acuática y el ser humano y un factor de deterioro de la calidad hídrica y ambiental. Los sedimentos ribereños, uno de los principales reservorios de estos elementos, actúan como fuentes secundarias de contaminación de los cuerpos de agua, por lo que resulta importante evaluar la concentración de metales como una herramienta que permite rastrear el origen de los contaminantes en el medio y predecir los impactos que pueden producir en los ecosistemas acuáticos. En el presente trabajo se analizó por espectrofotometría de absorción atómica la concentración de $\mathrm{Cd}, \mathrm{Ag}$, Se, Sn, Ni, Cr, Cu, B, Zn, Hg, Ba, Pb, Mn, As y Al en los sedimentos superficiales del sector medio del río Pirro (Heredia, Costa Rica). Las concentraciones de estos elementos fueron muy elevadas para la mayoría de las sustancias analizadas en todos los puntos de muestreo seleccionados. Su distribución no fue homogénea, ni presentó un patrón geográfico marcadamente definido, pudiéndose encontrar altos niveles distribuidos a lo largo del transecto estudiado.

\section{Objetivo general}

- Evaluar en forma preliminar la concentración de metales pesados en los sedimentos superficiales del Río Pirro.

\section{Keywords}

Pirro River, heavy metals, sediments.

\begin{abstract}
Anthropogenic activities are important sources of heavy metals, which are dangerous for the aquatic biota and the human being and a factor of environmental and water quality deterioration. The sediments, one of the main reservoirs of these elements, act as secondary resources of contamination of the rivers; this is why it is important to evaluate the concentration of metals, as a tool that allows to track the origin of the contaminants in the environment and to predict their impact on the aquatic ecosystems. In the present study, the concentration of $\mathrm{Cd}$, $\mathrm{Ag}$, Se, $\mathrm{Sn}, \mathrm{Ni}, \mathrm{Cr}, \mathrm{Cu}, \mathrm{B}, \mathrm{Zn}, \mathrm{Hg}, \mathrm{Ba}, \mathrm{Pb}, \mathrm{Mn}, \mathrm{As}$ and $\mathrm{Al}$ was determined in the superficial sediments of the medium sector of Pirro River (Heredia, Costa Rica) by using atomic absorption spectrophotometry. The concentrations of these elements were very high for the majority of the elements analyzed in all sampling points. Its distribution was not homogeneous, nor it presented a define geographical pattern, since high levels were found along the entire riverbed.
\end{abstract}

\section{Objetivos específicos}

- Evaluar la relación existente entre la concentración de metales pesados en los sedimentos del Río Pirro con otros parámetros físicos y químicos relevantes.

- Proponer de forma preliminar las fuentes naturales y antropogénicas que podrían contribuir a la presencia de metales pesados en los sedimentos superficiales del Río Pirro. 


\section{Introducción}

Los sedimentos ribereños están constituidos principalmente por la fracción sedimentable, orgánica y mineral de los sólidos suspendidos y por los desechos celulares de la actividad bacteriana, producidos en la oxidación de compuestos orgánicos biodegradables que ingresan al cauce por aporte de aguas domésticas y escorrentía superficial (Del Castillo, 2008). El aumento en el caudal y los cambios en la dinámica fluvial de los cuerpos de agua superficial pueden afectar drásticamente la configuración de su lecho, así como la distribución del sedimento, las plantas y la fauna a lo largo del cauce, generando problemas en el establecimiento de modelos aplicados a la hidroquímica de las corrientes naturales (Ruiz, Echeandía \& Romero, 1996).

Muchos de los contaminantes que ingresan a un cuerpo de agua superficial, por vía natural o antropogénica, quedan retenidos en los sedimentos que se depositan en el fondo del cauce, causando efectos tóxicos sobre los sistemas acuáticos (Estreves et al., 1996; Bohn et al., 200 I). Estos depósitos tienen un gran valor científico para el estudio de estos ecosistemas, pues conservan un registro histórico del tipo de contaminación que ha tenido lugar en las zonas próximas a su recorrido, y al interaccionar con las corrientes de agua ponen en circulación los contaminantes retenidos, alterando la dinámica general de la masa de agua (Mariani y Pompêo, 2008). Así, variaciones de $\mathrm{pH}$, salinidad y de las propiedades redox de las corrientes de agua pueden provocar movilización y resuspensión de especies químicas acumuladas en los sedimentos, magnificando incluso su efecto tóxico, tal como es el caso de los metales pesados (Ruiz et al., 1996).

Los metales pesados provienen en gran medida de fuentes antropogénicas, como desechos domésticos, agrícolas e industriales, y constituyen un peligro para la biota acuática y el ser humano, así como un factor de deterioro ambiental. Estos elementos se acumulan principalmente en los sedimentos superficiales de los ríos, aunque pueden encontrarse concentraciones relativamente elevadas a una profundidad de I5 cm y guardan una estrecha relación con el tamaño de las partículas que constituyen el sedimento (limo, arcilla y arena) y con la cantidad de materia orgánica sedimentaria, alterando el equilibrio ecológico y biogeoquímico del ecosistema (Acosta et al., 2002).
Debido a que la dinámica sedimentaria de los ríos determina en gran medida la distribución de contaminantes y su disponibilidad en la columna de agua a lo largo del tiempo, el objetivo de esta investigación fue evaluar de forma preliminar la concentración de metales pesados en los sedimentos de la parte media del río Pirro, así como algunas propiedades físicas y químicas relacionadas con su distribución a lo largo del cauce.

\section{Materiales y métodos}

La recolección de las muestras de sedimentos se realizó durante el mes de marzo de 2008 (época seca), en tres puntos de muestreo ubicados a lo largo del sector medio del río Pirro, en el Cantón Central de Heredia (Cuadro I). En cada uno de los puntos descritos se preparó una muestra compuesta por 10 submuestras, recolectadas a lo ancho del cauce a una profundidad aproximada de $10 \mathrm{~cm}$ y siguiendo una distribución aleatoria. La recolección se realizó con tubos cilíndricos de PVC de $10 \mathrm{~cm}$ de diámetro y $15 \mathrm{~cm}$ de altura. Las muestras recolectadas se almacenaron en bolsas plásticas con cierre hermético, se secaron al aire y se protegieron de la luz por una semana. Una vez secas, se pasaron por un tamiz de 9 mesh (2 mm), para el análisis de textura, humedad y metales pesados, y por un tamiz de 60 mesh $(25 \mathrm{~mm})$ para el análisis de materia orgánica.

Cuadro I. Ubicación de los puntos de muestreo

\begin{tabular}{|c|c|c|c|}
\hline $\begin{array}{l}\text { Punto de } \\
\text { muestreo }\end{array}$ & Lugar & $\begin{array}{c}\text { Altura } \\
\text { (msnm) }\end{array}$ & $\begin{array}{c}\text { Localización en } \\
\text { coordenadas } \\
\text { geográficas }\end{array}$ \\
\hline \multirow{2}{*}{ I } & \multirow{2}{*}{ Santiago } & \multirow{2}{*}{1150} & $10^{\circ} 00^{\prime} \mid 2,02^{\prime \prime} \mathrm{N}$ \\
\hline & & & $84^{\circ} 06^{\prime} \mid 8,36^{\prime \prime} \mathrm{O}$ \\
\hline \multirow{2}{*}{2} & \multirow{2}{*}{ Uriche } & \multirow{2}{*}{1125} & $09^{\circ} 59^{\prime} 51,05^{\prime \prime} \mathrm{N}$ \\
\hline & & & $84^{\circ} 06^{\prime} 24,05^{\prime \prime} \mathrm{O}$ \\
\hline \multirow{2}{*}{3} & \multirow{2}{*}{ La Puebla } & \multirow{2}{*}{1100} & $09^{\circ} 59^{\prime} 42,47^{\prime \prime} \mathrm{N}$ \\
\hline & & & $84^{\circ} 06^{\prime} 43,98^{\prime \prime} \mathrm{O}$ \\
\hline
\end{tabular}

Los procedimientos analíticos empleados para el análisis de los sedimentos se resumen en el Cuadro 2. El pretratamiento de las muestras y los análisis de humedad, materia orgánica y textura se realiza- 
Cuadro 2. Métodos analíticos empleados en el análisis de los parámetros físicos y químicos en muestras de sedimentos

\begin{tabular}{|c|c|c|c|}
\hline Parámetro & Unidades & Método & Descripción \\
\hline Humedad & $\mathrm{mg} / \mathrm{kg} \mathrm{H} \mathrm{H}_{2} \mathrm{O}$ & Gravimétrico & Secado de la muestra a $103-105^{\circ} \mathrm{C}$ por 24 horas. \\
\hline $\begin{array}{l}\text { Materia } \\
\text { orgánica }\end{array}$ & $\% \mathrm{MO}$ & Método REDOX & $\begin{array}{l}\text { Digestión con dicromato de potasio de la materia orgánica } \\
\text { presente en la matriz y posterior valoración del dicromato en } \\
\text { exceso en la muestra utilizando una solución de hierro (III) y } \\
\text { difenilamina como indicador. }\end{array}$ \\
\hline Textura & $\begin{array}{l}\% \text { arena } \\
\% \text { arcilla } \\
\% \text { limo }\end{array}$ & Hidrómetro Bouyoucos & $\begin{array}{l}\text { Dispersión de los agregados del suelo con una solución de } \\
\text { oxalato de sodio en medio básico y medición de la cantidad de } \\
\text { sólidos que se encuentran en suspensión a una temperatura de } \\
\text { referencia de } 20^{\circ} \mathrm{C} \text {. }\end{array}$ \\
\hline Metales & $\mathrm{mg} / \mathrm{kg}$ metales & $\begin{array}{l}\text { Espectrofotometría de } \\
\text { absorción atómica }\end{array}$ & $\begin{array}{l}\text { Digestión con ácido nítrico y medición espectrofotométrica } \\
\text { por absorción atómica acoplado a un equipo de generación de } \\
\text { hidruros para el análisis de mercurio. }\end{array}$ \\
\hline
\end{tabular}

ron conforme con lo planteado en el Manual de Prácticas de Química del Suelo (Leandro, 2007), mientras que el análisis de la concentración de metales pesados se realizó siguiendo la metodología AOAC (2006) para muestras de suelo.

\section{Resultados y discusión}

Los valores obtenidos para el contenido de materia orgánica y el análisis de textura se resumen en el Cuadro 3. Para todas las muestras analizadas, se encontró una alta proporción de arena en su composición, mientras que las fracciones arcillosa y limosa se hallaron en menor cantidad. La predominancia de la fracción arenosa sobre la arcillosa puede deberse a la topografía irregular del terreno así como a procesos erosivos y sedimentarios del lecho. Además, en la época seca, cuando el fenómeno de sedimentación supera al de mezcla, la fracción fina que se deposita sobre la gruesa es más susceptible al arrastre por las corrientes de agua, lo cual, suma- do a la disminución en el aporte de materiales de las laderas del río, permite que aumente la proporción de materiales gruesos en el sustrato (Leandro, 2006; Pérez et al., 2006).

En cuanto a la fracción orgánica del sedimento, se observó que para todos los puntos de muestreo evaluados el contenido promedio fluctuó entre 2, I I y $2,71 \%$, valores característicos de suelos volcánicos de reciente formación y de textura gruesa (andisoles), como los que se encuentran en los sitios en estudio (Pérez, 2007).

De los resultados obtenidos se deduce que la fracción más abundante en los sedimentos extraídos es la inorgánica (hidróxidos de hierro y manganeso, minerales de la arena y carbonatos), mientras que la fracción orgánica, compuesta por ácidos y materiales húmicos y biológicos como algas y bacterias floculadas, se encuentra en menor proporción. La naturaleza poco orgánica de los sedimentos y el pH del agua tienen una gran incidencia en la movilidad

Cuadro 3. Contenido promedio de materia orgánica y textura en los sedimentos

\begin{tabular}{|c|c|c|c|c|c|}
\hline \multirow{2}{*}{ Punto de muestreo } & \multirow{2}{*}{$\begin{array}{l}\mathrm{pH} \text { del agua } \\
\quad( \pm 0,0 \mathrm{I})\end{array}$} & \multirow{2}{*}{$\begin{array}{c}\text { Materia orgánica }( \pm 0,08 \\
\% \mathrm{~m} / \mathrm{m})\end{array}$} & \multicolumn{3}{|c|}{ Granulometría } \\
\hline & & & $\begin{array}{c}\text { Arena } \\
( \pm 0,1 \%)\end{array}$ & $\begin{array}{l}\text { Arcilla } \\
( \pm 0,1 \%)\end{array}$ & $\begin{array}{l}\text { Limo } \\
( \pm 0,1 \%)\end{array}$ \\
\hline Santiago & 7,05 & 2,71 & 84,1 & 12,9 & 3,0 \\
\hline Uriche & 6,98 & 2,61 & 83,7 & 10,3 & 6,1 \\
\hline La Puebla & 7,08 & 2,11 & 91,6 & 6,6 & 1,9 \\
\hline
\end{tabular}


y biodisponibilidad de los metales pesados en la columna de agua, ya que al disminuir el contenido de material orgánico en el sedimento también se reduce su capacidad de acumular metales, quedando estos disponibles en disolución, lo cual facilita su transporte a lo largo del cauce.

En la Figura I se muestran los metales que presentaron la menor concentración en los sedimentos extraídos. Se identificó un comportamiento poco variable en los tres puntos de muestreo para el $\mathrm{Cr}$, $\mathrm{Sn}, \mathrm{Ni}$ y Se, con valores comprendidos entre 10-44 $\mathrm{mg} / \mathrm{kg}$; mientras que para el $\mathrm{Pb}$ se obtuvieron concentraciones relativamente variables, con valores entre $19-44 \mathrm{mg} / \mathrm{kg}$ y concentraciones bajas de $\mathrm{Ag}$ y Cd, entre 0,26-2,2 mg/kg.

Las concentraciones de níquel encontradas fueron mayores en Santiago $(37 \pm \mathrm{I} \mathrm{mg} / \mathrm{kg})$, seguido de La Puebla $(3| \pm| \mathrm{mg} / \mathrm{kg})$, y presentaron su mínimo en Uriche $(29 \pm \mathrm{I} \mathrm{mg} / \mathrm{kg})$, aunque no existe diferencia estadísticamente significativa entre los valores. Dichos resultados concuerdan con los datos de textura reportados, pues el níquel tiende a acumularse en la fracción fina del sedimento, y por tanto los puntos donde el porcentaje de arcilla fue mayor, la concentración de Ni aumentó y viceversa. Asimismo, la presencia de níquel en los sedimentos estudiados puede deberse al ingreso al cauce de desechos no ordinarios, como electrodomésticos y baterías, que emplean aleaciones de níquel en su fabricación, y a productos líquidos usados en la industria metalmecánica, como aceites, pinturas y lubricantes procedentes del lavado de calles por la lluvia o que ingresan en forma directa al cauce (Marrugo et al., 2006).

La mayor concentración de estaño se registró en Uriche, con $38 \pm 1 \mathrm{mg} / \mathrm{kg}$, mientras que en los puntos Santiago y La Puebla se presentaron concentraciones similares de $25 \pm 1$ y $24 \pm 1 \mathrm{mg} / \mathrm{kg}$, respectivamente. Estos valores pueden deberse al ingreso de aguas residuales domésticas con altos contenidos de jabón y detergentes en cuya formulación se incluyan compuestos inorgánicos de estaño, residuos sólidos como plásticos y aguas de riego con plaguicidas u otros insumos agrícolas que contengan sales de estaño. De hecho, el ingreso de estaño a cuerpos de agua superficial está directamente relacionado con la actividad humana, pues son muy pocos los procesos geológicos que lo aportan naturalmente (ATSDR', 2003).

En cuanto a la concentración de cromo, Santiago fue el punto que mayor concentración registró (44 I mg/kg), seguido de La Puebla $(37 \pm$ I mg/ $/ \mathrm{kg}$ ) y Uriche $(34 \pm 1 \mathrm{mg} / \mathrm{kg})$. La entrada del cromo al agua puede deberse a procesos naturales y actividades antropogénicas. La precipitación por lluvias de compuestos de cromo que se encuentran en el aire formando parte de pequeñas partículas de polvo producto de la quema de carbón y derivados del

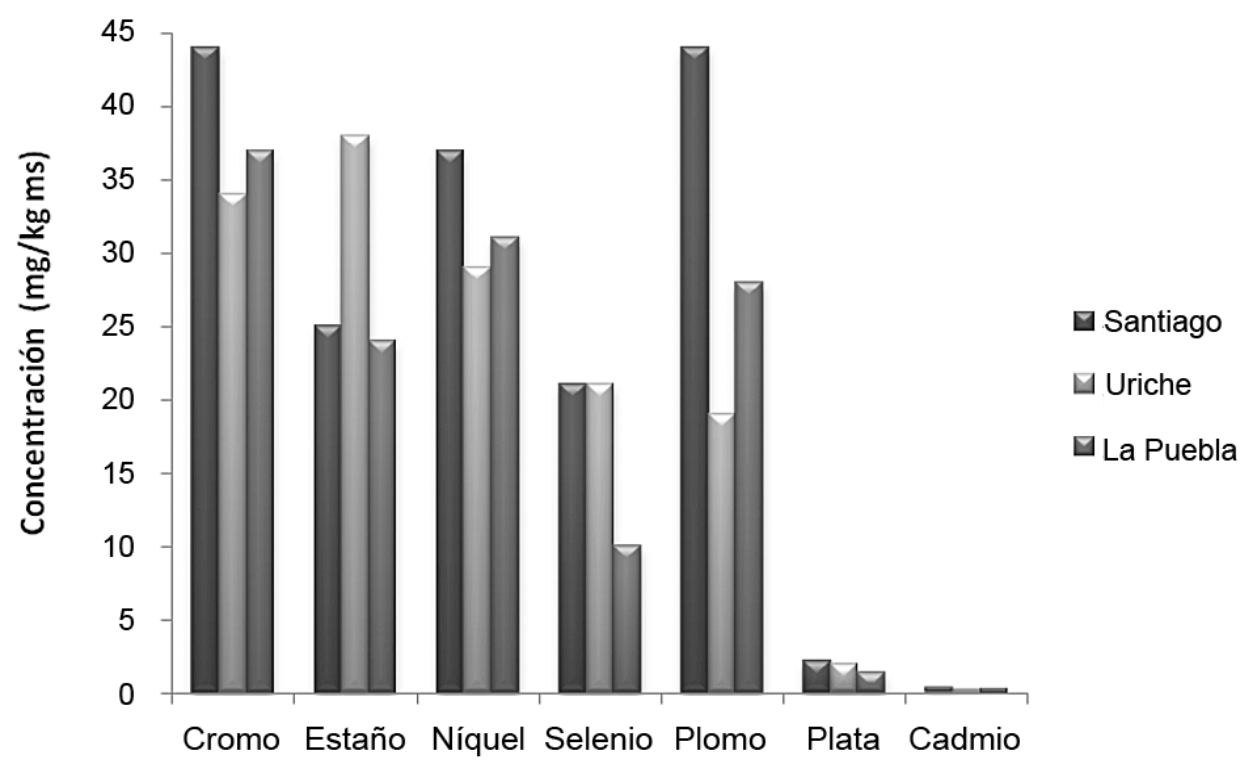

Figura I. Concentración de Cr, Sn, Ni, Se, Pb, Ag y Cd en los sedimentos recolectados en el río Pirro. 
petróleo, contribuye al aumento de la concentración de cromo en las aguas superficiales. Igualmente, el curtido de cueros y la industria textil, como también la manufactura de colorantes y pigmentos, pueden descargar cromo (III) y cromo (VI) a los cuerpos de agua. Sin embargo, como la actividad industrial de este tipo no se desarrolla en la parte superior y media de la microcuenca del río Pirro, se podría pensar que la presencia de cromo en los sedimentos se debe principalmente a procesos naturales como precipitación, evaporación, escurrimiento y vulcanismo (ATSDR, 2000).

En los sedimentos extraídos de los puntos Santiago y Uriche se encontró la máxima concentración de selenio, correspondiente a un valor de $21 \pm 1 \mathrm{mg} /$ $\mathrm{kg}$. Los aportes pudieron deberse principalmente a fuentes naturales como desgaste de suelos y rocas cercanas a la ribera del río, precipitación por lluvia de compuestos de selenio presentes en el aire y emisiones de la actividad volcánica. Aunque también debe considerarse la intervención humana a través del drenaje de aguas de regadío, sobre todo en los puntos cercanos a áreas de cultivos como Santiago, que se ubica en la proximidades de campos cafetaleros (ATSDR², 2003).

Las concentraciones de plomo obtenidas se encontraron en un rango de $19 \pm 1-44 \pm 1 \mathrm{mg} / \mathrm{kg}$, valores considerablemente mayores que los reportados por Wilcke et al. (2000) en sedimentos provenientes de suelos volcánicos (andisoles) de Costa Rica, los cuales no superan los $7,3 \mathrm{mg} / \mathrm{kg}$. La presencia de tales cantidades de plomo en los sedimentos pudo deberse al uso de fertilizantes y plaguicidas en áreas de cultivo próximas a la ribera del río, que ingresan al cauce por escorrentía superficial; a la acumulación de residuos de combustibles fósiles que contienen plomo y a la descarga de aguas de escorrentía de centros urbanos provenientes del lavado de calles y aceras impregnadas con aceites, pinturas y combustible. De hecho, los niveles de plomo más altos en aguas o sedimentos se presentan en las áreas más cercanas a carreteras (Navarro \& Sabater, 2004).

La concentración de plata en el sedimento fue muy baja en los tres puntos de muestreo estudiados. Se encontraron valores entre I,4 $\pm 0,1$ y 2,2 $\pm 0,1 \mathrm{mg} / \mathrm{kg}$; y su presencia se debe primordialmente a procesos naturales como la erosión de rocas por acción del viento y el agua, escorrentía superficial y precipitación atmosférica, que aportan pequeñas concentraciones del elemento al cuerpo de agua superficial.

En la Figura I se puede observar que los niveles de cadmio encontrados no superaron el valor

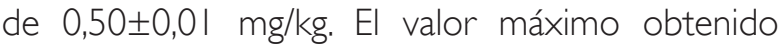
correspondió a $0,35 \pm 0,01 \mathrm{mg} / \mathrm{kg}$ para el punto

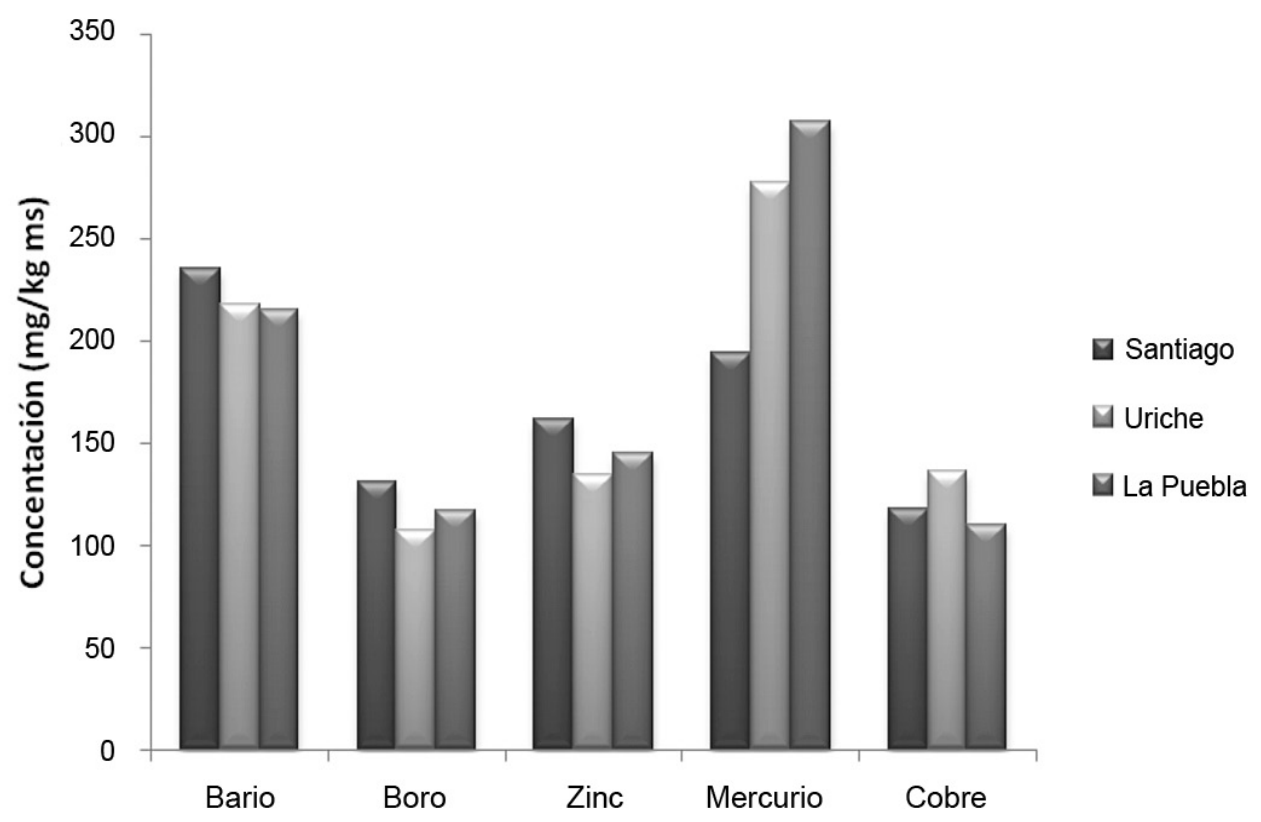

Figura 2. Concentración de Ba, B, Zn, Hg y Cu en los sedimentos recolectados en el río Pirro 
Santiago, mientras que en Uriche y La Puebla se encontraron valores de 0,26 $\pm 0,0$ I y 0,3 I $\pm 0,0$ I mg/ $\mathrm{kg}$, respectivamente. Aunque son muchas las fuentes que pueden aportar cadmio al cuerpo de agua en estudio, las concentraciones de este elemento en los sedimentos son bajas, debido a que las aguas poseen un $\mathrm{pH}$ neutro que impide que las sales de cadmio precipiten y se adsorban sobre las partículas del sedimento. Ello favorece su permanencia en el agua en forma disuelta, biodisponible para las plantas y animales que se encuentran en contacto con el medio, potencializando su efecto tóxico (Marrugo et al., 2006; Pérez et al., 2006; ATSDR, 2008).

En la Figura 2 se muestran los metales y no metales que presentaron mayor concentración en los sedimentos extraídos. Se debe resaltar la similitud en los resultados obtenidos en los tres puntos de muestreo para bario, boro, zinc y cobre, con valores comprendidos entre $107-235 \mathrm{mg} / \mathrm{kg}$. La concentración de mercurio en los sedimentos varió significativamente entre los diferentes puntos de muestreo en un rango de $194 \pm 10$ a $307 \pm 10 \mathrm{mg} / \mathrm{kg}$.

La concentración de bario obtenida en los puntos Santiago, Uriche y La Puebla fue de 235 \pm 1 0, $218 \pm 10$ y $215 \pm 10 \mathrm{mg} / \mathrm{kg}$, respectivamente. La presencia de este metal parece deberse principalmente a procesos erosivos y depósitos minerales en el fondo de los ríos. Aunque debe considerarse el aporte antropogénico por aguas residuales domésticas y de talleres mecánicos, cargadas de aceites, combustibles o pinturas, con aditivos de bario. Igualmente, cauces contaminados con desechos sólidos como llantas y botellas de vidrio pueden poner en circulación en la masa de agua cantidades pequeñas de bario, las que, dependiendo del $\mathrm{pH}$, precipitarán como sulfatos y carbonatos, incorporándose a los sedimentos (ATSDR', 2007).

Las concentraciones de boro medidas en los sedimentos de los puntos Santiago, Uriche y La Puebla fueron de $131 \pm 10,107 \pm 10$ y $117 \pm 10 \mathrm{mg} / \mathrm{kg}$, respectivamente, mientras que las concentraciones de zinc, en los mismos puntos, fueron ligeramente mayores, con valores de $162 \pm 10,134 \pm 10$ y $145 \pm 10$ $\mathrm{mg} / \mathrm{kg}$, respectivamente. El punto Santiago fue el que mayor concentración de estos elementos presentó, aunque no existe diferencia estadísticamente significativa entre los valores obtenidos de cada elemento para los diferentes puntos de muestreo evaluados.
La presencia de boro y zinc se debe principalmente al uso de plaguicidas y de fertilizantes en suelos de zonas cercanas al cauce receptor, que luego pasan al río por escorrentía superficial en época de lluvias o a través de las aguas de riego. El boro también puede ser liberado como consecuencia de la erosión natural de suelos y rocas; mientras que el zinc puede ingresar por precipitación atmosférica o a través de la quema de desechos sólidos en lotes baldíos cercanos al río (ATSDR, 1992; ATSDR, 2005).

La concentración de cobre superó los $100 \pm 10$ mg/ $\mathrm{kg}$ en los tres puntos de muestreo evaluados, lo que coincide con los valores de $136 \mathrm{mg} / \mathrm{kg}$ de Cu reportados por Wilcke et al. (2000) para los sedimentos provenientes de suelos andisoles de origen volcánico destinados a áreas de cultivo de café en Costa Rica, como los incluidos en el presente estudio.

La llegada del cobre al medio acuático, y posteriormente a los depósitos sedimentarios del río, va ligada a usos diversos en la agricultura y la industria. Por tanto, su ingreso al cuerpo superficial en estudio puede deberse al empleo de fertilizantes y fungicidas formulados a partir de $\mathrm{Cu}$ en las áreas de cultivo de la parte alta de la microcuenca, al ingreso de lixiviados provenientes de depósitos de basura cercanos a las riberas del río, que por lo general se ubican en lotes baldíos utilizados como botaderos a cielo abierto, a la quema de desechos sólidos en las cercanías del río, al ingreso de aguas residuales domésticas, al uso de combustibles fósiles y a fuentes naturales como vulcanismo, vegetación en descomposición e incendios forestales (ATSDR, 2004; Navarro \& Sabater, 2004).

Las concentraciones de mercurio en los sedimentos evaluados presentaron una tendencia creciente al disminuir la altitud del punto de muestreo. Así, la mayor concentración se reportó en el punto La Puebla, con un valor de $307 \pm 10 \mathrm{mg} / \mathrm{kg}$. El mercurio presente en los sedimentos pudo provenir tanto de fuentes naturales como de la actividad humana. La erosión de rocas y suelos y la actividad volcánica pueden introducir pequeñas cantidades en la atmósfera, donde se transforma en otras formas de mercurio que pueden ser transportadas al agua y los sedimentos por la lluvia. Sin embargo, aun cuando el aporte proveniente de fuentes naturales se debe considerar, dos tercios de la contaminación mundial por mercurio en agua y sedimentos se debe a contaminación antropogénica (Marrugo et al., 2006). El 
uso de combustibles fósiles, abonos, fertilizantes y fungicidas y la disposición en los cauces de desechos sólidos como baterías, interruptores eléctricos, aparatos electrónicos y termómetros, pueden introducir grandes cantidades de mercurio a los cuerpos de agua superficial, así como la quema incontrolada de desechos municipales en los lotes baldíos y las aguas residuales provenientes del lavado de calles y aceras cargadas de aceites y combustibles (ATSDR, 1999).

En la Figura 3 se muestran los resultados obtenidos para manganeso, arsénico y aluminio. Estos valores superaron los $458 \mathrm{mg} / \mathrm{kg}$, y representan las especies metálicas de más abundancia en los sedimentos del área de estudio.

Los valores obtenidos para el manganeso son típicos en sedimentos derivados de suelos volcánicos y su presencia se debe principalmente a procesos naturales como erosión de rocas y material parental (Wilcke et al., 2000). La actividad humana tiene poca incidencia sobre la concentración de manganeso presente en el cauce y en los fondos sedimentarios y responde principalmente a operaciones muy específicas de minería y plantas procesadoras de hierro y acero, actividades que no se desarrollan en la microcuenca analizada.

Por otro lado, los altos contenidos de arsénico son comunes en sedimentos que poseen concentraciones importantes de otros metales como cobre y plomo y su presencia pudo deberse, entre otras cosas, al uso de plaguicidas en áreas de cultivo, aunque el tipo de agricultura que se desarrolla en la zona pareciera no favorecer su empleo. También, deben considerarse otros aportes que aumentan su concentración, entre ellos, la disposición de desechos sólidos en el cauce como baterías y aparatos electrónicos, la quema de combustibles fósiles y la combustión de desechos sólidos municipales en lotes baldíos próximos a la ribera del río (ATSDR², 2007).

Las concentraciones de aluminio superaron los $48 \pm \mathrm{l} \mathrm{g} / \mathrm{kg}$, no obstante, estos valores son comunes en suelos andisoles, pues forman parte fundamental de las unidades alófanas de este tipo de suelos (Wilcke et al., 2000).

Finalmente, resulta importante mencionar que las principales vías de transporte de los metales pesados desde el suelo y el aire hacia el agua, son la escorrentía superficial, la percolación y la precipitación atmosférica. La facilidad con que los contaminantes pasan del suelo al agua, y posteriormente a los fondos sedimentarios, dependerá de las propiedades físicas y químicas de las unidades implicadas, además de la composición química en que se encuentra el contaminante y la geomorfología del área en estudio. Otros factores que pueden aumentar las concentraciones de metales pesados en los sedimentos son las fuentes no puntuales de origen natural o antropogénico.

\section{Conclusiones}

Este trabajo pone de manifiesto un potencial y grave problema ambiental, derivado del alto contenido de metales pesados presentes en los sedimentos del río Pirro. El vertido de aguas residuales sin pre-
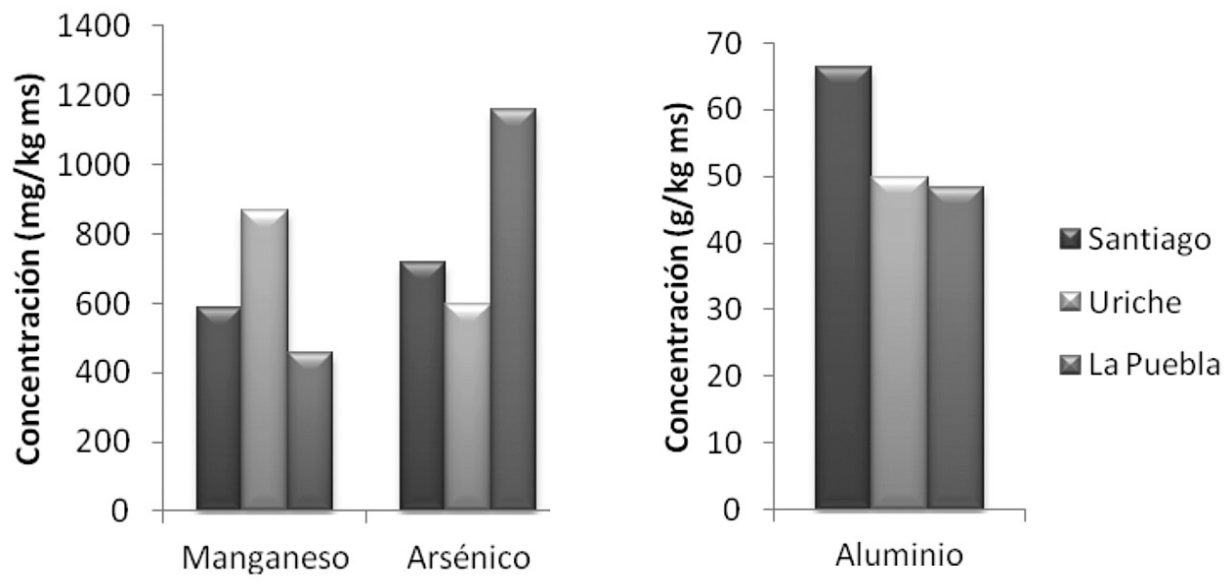

Figura 3. Concentración de Mn, As y Al en los sedimentos recolectados en el río Pirro 
vio tratamiento, la infiltración de aguas negras y el desarrollo de actividades agrícolas, metalúrgicas y metalmecánicas en la parte alta de la microcuenca han favorecido su incorporación e incremento en los lechos arenosos del cauce. Sin embargo, la principal amenaza de este tipo de contaminación no reside en su acumulación en la fracción sedimentable sino en los mecanismos de sorción-desorción y precipitación-disolución, dependientes de las condiciones físicas y químicas del río, que provocan su resuspensión en la columna de agua dejándolos biodisponibles para la vegetación acuática. El mayor peligro del aumento en la concentración de metales pesados en los lechos sedimentarios es primordialmente su incorporación a las cadenas tróficas, su magnificación y bioacumulación en los organismos, y en su dificultad de eliminación.

\section{Recomendaciones}

Para que la evaluación de metales pesados en los sedimentos sirva como una herramienta estandarizada para la localización de las fuentes de contaminación dentro de una unidad hidrológica, se recomienda establecer valores de referencia de línea base dentro del área de estudio, con el objetivo de diferenciar si las contribuciones son de origen natural o antropogénico. Estos valores de referencia se pueden obtener de zonas muy poco pobladas, o bien del análisis de los sedimentos en los horizontes B o C, los cuales debido a la profundidad estarán menos influenciados por los procesos de erosión y contaminación.

Además, sería muy valioso complementar el análisis de metales pesados en el sedimento con el análisis de metales pesados en la columna de agua, para determinar su removilización y biodisponibilidad.

\section{Agradecimientos}

Los autores expresan su agradecimiento a la Oficina Ejecutora de la Universidad Nacional para el préstamo del Banco Centroamericano de Integración Económica (BCIE) en la persona del Arq. Carlos Segura Murillo por el apoyo económico y estratégico suministrado. También, al Laboratorio de Manejo del Recurso Hídrico y al Laboratorio de Análisis y Servicios Químicos de la Universidad Nacional (LASEQ) en la persona del M.Sc. Efraín Solís Montiel por su gran apoyo en el proyecto.

\section{Bibliografía}

Acosta, V., Lodeiros, C., Senior,W. \& Martínez, G. (2002). Niveles de metales pesados en sedimentos superficiales en tres zonas litorales de Venezuela. Interciencia 27(I2): 686-690.

AOAC (2006). Official Methods of Analysis of AOAC International. 17 ed. Official Method 986.15. Gaithersburg, MD, USA: AOAC INTERNATIONAL.

ATSDR (Agency forToxic Substances \& Disease Registry). ( 1992). Reseña toxicológica del boro. Atlanta, GA: Departamento de Salud y Servicios Humanos de EE. UU., Servicio de Salud Pública, I I p.

ATSDR (Agency for Toxic Substances \& Disease Registry). (1999). Reseña toxicológica del mercurio y compuestos del mercurio. Atlanta, GA: Departamento de Salud y Servicios Humanos de EE. UU., Servicio de Salud Pública, I 3 p.

ATSDR (Agency forToxic Substances \& Disease Registry). (2000). Reseña toxicológica del cromo. Atlanta, GA: Departamento de Salud y Servicios Humanos de EE. UU., Servicio de Salud Pública, 10 p.

ATSDR' (Agency for Toxic Substances \& Disease Registry). (2003). Reseña toxicológica del estaño y compuestos de estaño. Atlanta, GA: Departamento de Salud y Servicios Humanos de EE. UU., Servicio de Salud Pública, I 5 p.

ATSDR $^{2}$ (Agency for Toxic Substances \& Disease Registry). (2003). Reseña toxicológica del selenio. Atlanta, GA: Departamento de Salud y Servicios Humanos de EE. UU., Servicio de Salud Pública, 8 p.

ATSDR (Agency forToxic Substances \& Disease Registry). (2004). Reseña toxicológica del cobre. Atlanta, GA: Departamento de Salud y Servicios Humanos de EE. UU., Servicio de Salud Pública, 10 p.

ATSDR (Agency forToxic Substances \& Disease Registry). (2005). Reseña toxicológica del zinc. Atlanta, GA: Departamento de Salud y Servicios Humanos de EE. UU., Servicio de Salud Pública, 9 p.

ATSDR' (Agency for Toxic Substances \& Disease Registry). (2007). Reseña toxicológica del bario y compuestos de bario. Atlanta, GA: Departamento de Salud y Servicios Humanos de EE. UU., Servicio de Salud Pública, 14 p.

ATSDR $^{2}$ (Agency for Toxic Substances \& Disease Registry). (2007). Reseña toxicológica del plomo. Atlanta, GA: Departamento de Salud y Servicios Humanos de EE. UU., Servicio de Salud Pública, 12 p.

ATSDR (Agency for Toxic Substances \& Disease Registry). (2008). Reseña toxicológica del cadmio. Atlanta, GA: Departamento de Salud y Servicios Humanos de EE. UU., Servicio de Salud Pública, 8 p.

Bohn, H., McNeal, B. \& O Connor, G. (200 I). Soil Chemistry. 2da ed. Canadá: John Wiley \& Sons, Inc, 34I p.

Del Castillo, I. (2008). Teoría de la depuración biológica. Clase magistral impartida en el curso "Reutilización y tratamiento de aguas residuales". Maestría en Gestión y Estudios Ambientales, Universidad Nacional, Heredia, Costa Rica, $15 \mathrm{p}$. 
Esteves, J., Gil, M. \& Harvey, M. (1996). Evaluación de la contaminación por metales en sedimentos y materiales en suspensión de la cuenca Turbio-Gallegos. Informe Técnico $N^{0} 12$ del Plan de Manejo Integrado de la Zona Costera Patogónica. Puerto Madryn, Argentina, $21 \mathrm{p}$.

Leandro, H. (2006). Indicadores de calidad del agua como instrumentos para la gestión de la microcuenca IV del Río Virilla. Tesis de Licenciatura en Química Industrial. Facultad de Ciencias Exactas y Naturales, Universidad Nacional, Heredia, 101 p.

Leandro, H. (2007). Manual del Laboratorio de Química de Suelos. Escuela de Química, Facultad de Ciencias Exactas y Naturales, Universidad Nacional, Heredia, 62 p.

Mariani, C. \& Pompêo, M. (2008). La calidad del sedimento: La contaminación por metales puede ser una amenaza para los seres vivos. Revista Ciencia Hoy, 18(107): 48-53.

Marrugo, J., Lans, E., Doria, G., Bello, L., Castillo, M., Cortes, F. \& Pinedo, J. (2006). Impacto ambiental por contaminación con níquel, mercurio y cadmio en aguas, peces y sedimentos en la cuenca del Río San Jorge, en el Departamento de Córdoba. Universidad de Córdoba, Centro de Investigaciones CIUC. Monteira, Colombia, 109 p.
Navarro, E. \& Sabater, S. (2004). Contaminación de los ríos por metales pesados. Investigación y Ciencia 336, 26-31.

Pérez, M., Martínez, G. \& Fermín, I. (2006). Biodisponibilidad de metales trazas en sedimentos superficiales del ecosistema Laguna Costero Bocaripo-Chacopata (Península de Araya, Esta de Sucre). Universidad de Oriente, Boletín del Instituto Oceanográfico de Venezuela 45(2): 8I-91.

Pérez, O. (2007). Guía de recomendaciones de nitrógeno para el cultivo de caña de azúcar en la zona cañera de Guatemala. Programa Agronómico CENGICAÑA, Guatemala, 5 p.

Ruiz, E.; Echenandía, A. \& Romero, F. (1994). Relaciones entre agua y sedimento en río de origen torrencial. Limnética, IO(I), 101-107.

Wilcke, W.; S. Kretzschmar; M. Bundt; G. Saborío \& W. Zech. 2000. Depth Distribution of Aluminium and Heavy Metals in soil of Costa Rican coffee cultivation area. J. Plant Nutri. Soil Sci, 163, 499-502.

Winkler, M. 1999. Tratamiento biológico de aguas de desecho. Editorial Limusa, S.A. de C.V., Sexta reimpresión, México, D.F., 338 